\title{
Long-Term Follow-Up of Denosumab Discontinuers with Multiple Vertebral Fractures in the Real-World: A Case Series
}

Authors

Liana Tripto-Shkolnik¹, 2, Yair Liel ${ }^{3}$, Naama Yekutiel ${ }^{4}$, Inbal Goldshtein², 4

\author{
Affiliations \\ 1 Division of Endocrinology, Diabetes and Metabolism, Chaim \\ Sheba Medical Center at Tel Hashomer, Ramat Gan, Israel \\ 2 Sackler Faculty of Medicine, Tel Aviv University, Tel Aviv, \\ Israel \\ 3 Ben-Gurion University of the Negev, Beer-Sheva, Israel \\ 4 Maccabitech Institute of Research and Innovation, \\ Maccabi Healthcare Services, Tel Aviv, Israel
}

Key words

osteoporosis, vertebral fractures, treatment discontinuation, denosumab discontinuation

received 19.09 .2020

accepted after revision $\quad 12.01 .2021$

published online 15.02 .2021

Bibliography

Horm Metab Res 2021; 53: 185-190

DOI 10.1055/a-1368-4218

ISSN 0018-5043

(c) 2021. Thieme. All rights reserved.

Georg Thieme Verlag KG, Rüdigerstraße 14,

70469 Stuttgart, Germany

Correspondence

Liana Tripto-Shkolnik MD

Division of Endocrinology, Diabetes and Metabolism,

Chaim Sheba Medical Center at Tel Hashomer

Ramat Gan

Israel

Tel: + 97252 6334348, Fax: + 97235305479

lianatrish@gmail.com

\section{ABSTRACT}

Denosumab discontinuation is associated with rapid reversal of bone turnover suppression and with a considerable increase in fracture risk, including a risk for multiple vertebral fractures (MVF). Long-term follow-up of patients who sustained MVF after denosumab discontinuation has not been reported. This case-series was aimed to provide a long-term follow-up on the management and outcome of denosumab discontinuers who initially presented with multiple vertebral fractures. Denosumab discontinuers were identified from a computerized database of a large healthcare provider. Baseline and follow-up clinical, laboratory, and imaging data were obtained from the computerized database and electronic medical records. The post-denosumab discontinuers MVF patients consisted of 12 women aged $71 \pm 12$. Osteoporotic fractures were prevalent before denosumab discontinuation in 6 of the patients. The majority received bisphosphonates before denosumab. MVF occurred $134 \pm 76$ days after denosumab discontinuation. The patients were followed for a median of 36.5 (IQR 28.2, 42.5) months after MVF. Two patients passed-away. Two patients suffered recurrent vertebral fractures. Following MVF, patients were treated inconsistently with denosumab, teriparatide, oral, and intravenous bisphosphonates, in various sequences. Two patients underwent vertebroplasty/kyphoplasty. This long-term follow-up of real-world patients with MVF following denosumab discontinuation reveals that management is inconsistent, and recurrent fractures are not uncommon. It calls for clear management guidelines for patients with MVF after denosumab discontinuation and for special attention to this high-risk group.

\section{Introduction}

Denosumab [1] is a fully-humanized monoclonal antibody against RANK-ligand, and a potent antiresorptive drug used for the treatment of osteoporosis [2], effective in increasing bone mineral density (BMD) and preventing osteoporotic fractures during, at least, 10 years of continuous treatment [3]. Denosumab is administered at 6 months intervals. Soon after its administration, bone resorption is markedly suppressed, as reflected by a decrease in bone turnover markers (BTM) [4]. Bone turnover suppression persists for 6 months and reverses rapidly thereafter [5]. If denosumab admin- istration is not reinstituted, a rebound acceleration of bone turnover occurs [6]. The pivotal FREEDOM trial demonstrated an increase of bone turnover rate after a median follow-up of 2.4 months following the would-be scheduled injection, associated with a decrease in BMD and increase in vertebral fracture rate, similar to the fracture rate observed in the placebo group [7, 8].

Since 2015, attention was drawn to the possibility that denosumab discontinuation could be linked to an increased risk for vertebral fractures, including multiple vertebral fractures (MVF) [916]. A mechanism involving rebound elevation of bone turnover 
and an increased osteoclastogenesis was proposed [17], as well as an accelerated targeted repair of accumulated microdamage [10]. A post-hoc analysis of data from the FREEDOM trial and its extension revealed that denosumab discontinuation was associated with a 6 -fold increase in the rate of vertebral fractures, approaching fracture rate in the placebo group, while the proportion of multiple vertebral fractures was 1.6-fold higher in the denosumab discontinuation group, more so in patients with prior vertebral fractures [18]. We obtained similar results, derived from a real-world setting (a large health provider's database), comparing denosumab discontinuers and persistent users, and identified chronic kidney failure and cerebrovascular disease as factors associated with fractures following denosumab discontinuation [1]. It has been shown that bone-resorption rate was attenuated in denosumab discontinuers treated with bisphosphonates (BP) before denosumab [19], but the evidence regarding such protection against rebound acceleration of bone turnover and fractures is still controversial $[9,15,16,20]$.

Bisphosphonates are the most recommended treatment modality post denosumab discontinuation [21]. Some controversy exists to whether a potent intravenous BP should be preferred or a more gradually acting oral medication, with several reports supporting oral [22, 23], and others intravenous route [24]. Denosumab treatment duration prior to discontinuation and individual fracture risk should be taken into consideration as well [21].

\section{Study objectives}

In the present report, we provide a unique long-term observation on the management and outcomes of patients with post-denosumab discontinuation MVF, initially identified in a previous study from a large Israeli healthcare provider's database [1].

\section{Materials and Methods}

The study was performed using longitudinal data from the Maccabi Healthcare Services (MHS) computerized database. MHS is the second largest healthcare provider and insurer in Israel serving approximately $25 \%$ of the population with a countrywide coverage. It maintains a central electronic database since 1998, including medical records, laboratory test results, imaging, diagnoses, medical procedures, referrals, prescriptions, and medication purchases. In order to be reimbursed by the comprehensive national healthcare insurance plan, denosumab should be mandatorily purchased in any of the pharm facilities associated with MHS and recorded in its database. Data collection for our core study [1] ended by June 2018, and the data collection for the current report ended by April 2020.

Patients were detected as previously described [1]. Denosumab initiators, with $>2$ consecutive medication purchases starting January 2012, were identified. Treatment discontinuation was defined as a refill gap of 3 months or more ( $>9$ months after the last denosumab dispensation). The date of an expected refill ( 6 months following the last refill) was considered the discontinuation date. Patients with MHS membership of fewer than 12 months pre- or 15 months post denosumab initiation date were excluded.

Major osteoporotic fractures were categorized according to the MHS osteoporosis registry, which includes vertebral, hip, distal forearm, and proximal arm fractures [25]. All vertebral fractures, including multiple vertebral fractures (MVF), were reviewed and adjudicated. Two or more vertebral fractures were classified as MVF.

Fractures were defined as "post discontinuation" if they occurred within 1 year from denosumab discontinuation.

Demographic and additional data was collected from electronic medical records and the MHS oncology, cardiovascular or cerebrovascular registries, including osteoporosis treatment before denosumab, data on traditional risk-factors for osteoporotic fractures: body mass index (BMI), bone mineral density (BMD), alcoholic beverages drinking habits, smoking, fracture history, secondary causes of osteoporosis (rheumatoid arthritis, chronic or ever exposure to systemic glucocorticoids). Medication possession ratio (MPR) of osteoporosis medications before denosumab treatment was calculated from the electronic medical records and expressed as a percentage of purchases per each year of treatment. Post-MVF osteoporosis treatments, treatment duration, recurrent vertebral fractures, and fatalities, were extracted. Recurrent vertebral fractures were considered "new" if they occurred > 12 months after the index MVF event.

Continuous variables are expressed as mean \pm standard deviation, or median and interquartile range (IQR). Categorical data are expressed as counts and percentages.

All procedures in this study followed the 1964 Helsinki declaration and its amendments. The study was approved by the MHS Ethics Committee.

\section{Results}

The core study included 1500 denosumab discontinuers [1]. Twelve female patients with MVF were identified among the discontinuers. The characteristics at denosumab initiation of the included patients are presented in $>$ Table 1. MVF events occurred a mean $3 \pm 1.2$ denosumab injections and $134 \pm 76$ days after denosumab discontinuation. Of the 12 patients who sustained MVF, 3 fractured 2 vertebrae and 8 fractured 3 vertebrae. In one case, the precise number of fractured vertebrae could not be determined.

- Table 2 presents the prior fractures and pre-denosumab medications details of the MVF patients. Osteoporotic fractures before denosumab treatment occurred in $6 / 12$ patients. All but one of the 12 described patients received other osteoporosis medications before denosumab. A total of 10/12 patients were pre-exposed to bisphosphonates. One patient $(\mathrm{P} 3)$ received strontium ranelate, and one patient (P10) received raloxifene. One patient (P7), completed a two years course of teriparatide 16 months before starting denosumab. Mean MPR for oral BPs was $67.8 \pm 14.7 \%$. Five patients with prior BP exposure had less than one-year washout of BP before starting denosumab treatment (grey-shaded rows). MPR in this subgroup was $69.8 \pm 15.7 \%$.

- Figure 1 presents the post-MVF management and clinical outcomes. The median follow-up duration after MVF was 36.5 (IQR 28.2-42.5) months. Six patients (P1-P4, P6, P9) resumed denosumab treatment at some point following MVF, two of them did not persist with the re-treatment (P3 and P6); one of them (P6) suffered an additional vertebral fracture. One patient (P4) died soon (1 month) following the resumed treatment. Four patients (P5, P7 and $\mathrm{P9}-10$ ) received teriparatide as a first medication following MVF, one of them (P9) was subsequently given an additional deno- 
- Table 1 Characteristics at denosumab initiation of patients with MVF.

\begin{tabular}{|c|c|}
\hline Characteristics & $\begin{array}{l}\text { Patients with MVF, } n=12 \\
\text { among DD }\end{array}$ \\
\hline Age, years, mean (SD) & $71(12)$ \\
\hline Female, \% & 100 \\
\hline Prior fractures, n (\%) & $6(50)$ \\
\hline Prior vertebral fractures, n (\%) & $4(33.3)$ \\
\hline Prior hip fractures, n (\%) & $1(8)$ \\
\hline Prior non-hip-non-vertebral fractures, $\mathrm{n}$ (\%) & $3(25)$ \\
\hline Prior Osteoporosis treatment, $\mathrm{n}(\%)$ & $11(91.6)$ \\
\hline $\begin{array}{l}\text { Denosumab injections prior to discontinua- } \\
\text { tion, mean (SD) }\end{array}$ & $3(1.2)$ \\
\hline Diabetes, n (\%) & $4(33)$ \\
\hline Rheumatoid arthritis, n (\%) & $2(16.6)$ \\
\hline Ever glucocorticoid use, $\mathrm{n}(\%)$ & $7(58.3)$ \\
\hline Current smoking, n (\%) & $2(16.6)$ \\
\hline Past smoking, $\mathrm{n}(\%)$ & 0 \\
\hline Femoral neck T-score, mean (SD) & $-2.34(0.8)$ \\
\hline Lumbar spine T-score, mean (SD) & $-2.42(1.9)$ \\
\hline $\mathrm{BMI}, \mathrm{kg} / \mathrm{m}^{2}$, mean (SD) & $26.4(3)$ \\
\hline eGFR, ml/min/1.73 m², mean (SD) & $73(27)$ \\
\hline Cancer, n (\%) & $3(25)$ \\
\hline Cardiovascular disease, $\mathrm{n}(\%)$ & $4(33)$ \\
\hline Cerebrovascular disease, n (\%) & $3(25)$ \\
\hline
\end{tabular}

MVF: Multiple vertebral fractures; DD: Denosumab discontinuers. sumab injection and was then switched to zoledronic acid. One patient $(\mathrm{P} 10)$ received oral alendronate, one patient $(\mathrm{P} 3)$ received oral risedronate alternating with denosumab, and one (P8) received risedronate for 6 months, was subsequently switched to zoledronic acid and then to teriparatide.

Two patients received no pharmacological treatment following MVF. One of those, died 14 months after the MVF event. Of note, two patients received teriparatide (P7) and risedronate (P8) for two months following denosumab discontinuation, before MVF occurred ( $\triangleright$ Fig. 1).

Two patients underwent vertebroplasty/kyphoplasty (P5 and P10), four and three months following the MVF event, respectively. In both cases the procedure was performed while on teriparatide and no additional vertebral fractures were recorded during 25 (P5) and 38 (P10) months of follow-up.

\section{Discussion and Conclusions}

We present patient-level data from a subgroup of patients with multiple vertebral fractures (MVF), derived from a large real-world cohort of patients monitored during one year for fractures following denosumab discontinuation. Our core study [1] demonstrated that any fracture, including MVF, occurred more frequently in patients who discontinued denosumab treatment than in persistent users, providing a real-world perspective on post denosumab discontinuation fractures. Data presented in the current report concerns patients with MVF and focused on existing osteoporosis risk factors, detailed prior treatment, fractures timing, and long-term follow-up for post-MVF treatments as well as clinical outcomes.

Remarkably, all our patients with MVF following denosumab discontinuation were women. This corresponds with the vast majority of the previously published cases on vertebral fractures follow-

- Table 2 Prior osteoporotic fractures and pre-denosumab osteoporosis treatment.

\begin{tabular}{|l|l|l|l|l|}
\hline Patient (P) & $\begin{array}{l}\text { Prevalent vertebral/hip } \\
\text { fractures }\end{array}$ & $\begin{array}{l}\text { Denosumab ini- } \\
\text { tiation year }\end{array}$ & $\begin{array}{l}\text { Prior osteoporosis treatment, years, } \\
\text { medication }\end{array}$ & $\begin{array}{l}\text { Medication possession ratio, oral } \\
\text { medications, average (\%) }\end{array}$ \\
\hline P1 & & 2013 & $1999-2007$, oBP & 59 \\
\hline P2 & 2015 & $2008-2015$, oBP & 92 \\
\hline P3 & & 2013 & $2006-2012$, oBP 2012-2013, Strontium & 92 \\
\hline P4 & Vertebral, hip & 2016 & $2004-2005$, oBP 2009-2012, oBP 2012, 2013, & 73 \\
\hline P5 & Vertebral & 2016 & 2014, IV Zoledronic acid & \\
\hline P6 & & 2014 & $2008-2014$, oBP & 58 \\
\hline P7 & Vertebral $x$ & 2015 & $2010-2011$, oBP 2012-2014, Teriparatide & 80 \\
\hline P8 & & 2015 & $2010-2014$, oBP & 50 \\
\hline P9 & & 2012 & $2000-2011$, oBP & 53 \\
\hline P10 & & 2014 & $2011-2012$, Raloxifene & 25 \\
\hline P11 & Vertebral & 2014 & 2013, oBP & NA \\
\hline P12 & & 2015 & $2004-2014$, oBP & 72 \\
\hline
\end{tabular}

oBP: Oral bisphosphonate; IV: Intravenous; NA: Not applicable. Grey-shaded rows: less than 365 days between BP treatment and denosumab initiation. 


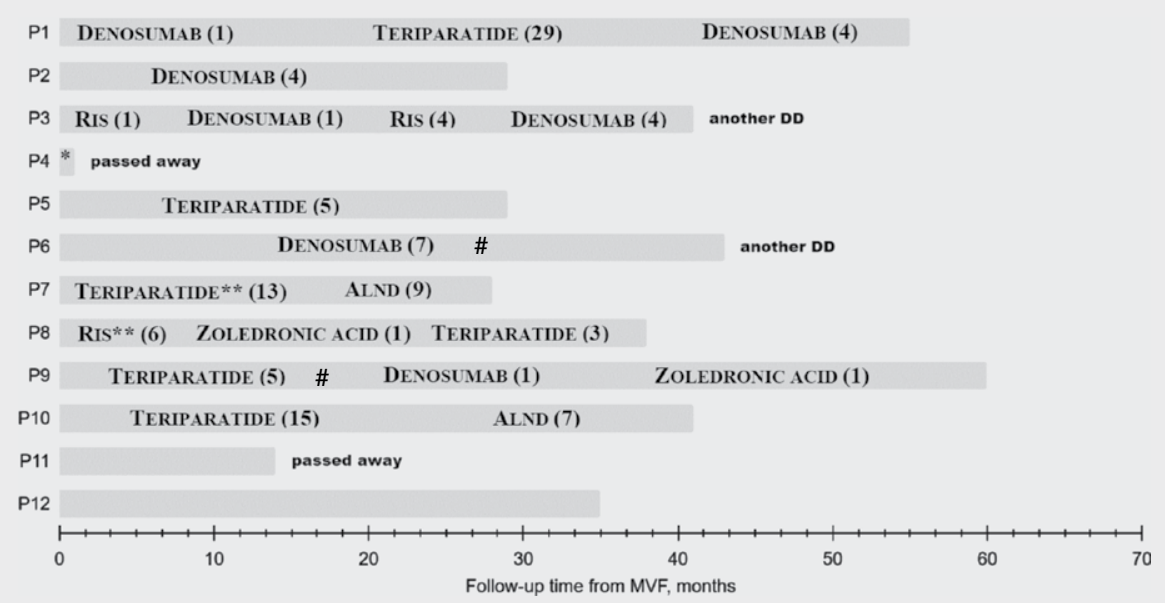

-Fig. 1 Treatment allocations and clinical outcomes post MVF. Post MVF Treatments: Medication (number of purchases); MVF- Multiple vertebral fractures, DD- Denosumab discontinuation; Ris: Risedronate; Alnd: Alendronate; ${ }^{*}$ :Denosumab (1); ${ }^{*}$ : MVF occurred two months after treatment initiation; \#: Recurrent vertebral fracture.

ing denosumab discontinuation $[15,16,26]$. We are aware of only two reported male patients with vertebral fractures following denosumab discontinuation, one of them with extremely low BMD, and the other with glucocorticoid-induced osteoporosis [27]. Osteoporotic fractures, although more prevalent in women, are reported in 1 in 3 men after 50 , and the prevalence of vertebral fractures in men $>65$ years old approaches half the rate among women [28]. The reason for the female dominance among the reported cases with vertebral fractures following denosumab discontinuation is not clear. It could be due to a differentially higher bone turnover rate in women during the first decade following menopause, which is not present in men due to residual exposure to sex hormones [29], or due to structural and/or biomechanical differences between male and female vertebrae [30]. The most plausible reason is the paucity of denosumab prescriptions and anti-osteoporosis medications at large, in men in general [31]. In our core study, among 1500 denosumab discontinuers, only 8 percent were male [1].

Prevalent VFs were documented in one-third of our MVF patients, which is not different than what is expected in postmenopausal women, in whom prevalence of vertebral fractures is reported to be between 20 and $29 \%$ in those over 75 [32, 33]. Nevertheless, it should be noted that this historical prevalence rates include asymptomatic vertebral deformities (about 2/3 of cases), while most prevalent fractures in our core study were identified via coded diagnoses and were therefore, in most part, clinically evident, suggesting that vertebral fractures in our series could be more prevalent than in the general population. The higher incidence of MVF in a subgroup with prevalent vertebral fractures among denosumab discontinuers was previously emphasized [18], indicating increased baseline fragility of the vertebrae before denosumab treatment, and implying that allocation to denosumab treatment may comprise a patients' selection bias.

It has been suggested that the rebound increase in bone resorption following denosumab discontinuation was less pronounced in patients treated with bisphosphonates before denosumab treatment [19], and MVF seemed to be less common [15], however, this is not necessarily generalizable. In a series of high-risk osteoporotic patients with vertebral fractures following denosumab discontinuation reported previously, all but one with MVF, long-term bisphosphonate treatment before receiving denosumab was observed [16]. In the present study, a majority (10/12) of the MVF patients were treated with oral and/or intravenous bisphosphonates for prolonged periods before the initiation of denosumab treatment, with an MPR close to $70 \%$. This suggests that bisphosphonate exposure before denosumab treatment has only a limited protective effect, if any, against MVF following denosumab discontinuation. The prevailing concept of over-suppression of bone turnover during long-term oral bisphosphonate treatment has recently been challenged following observations that a considerable proportion of the patients on long-term oral bisphosphonates had a high level of bone resorption [34].

The number of fractured vertebrae per patient in our case-series is somewhat lower than previously reported [15, 16, 35]. This could be due to a selection bias in previous case series of patients with high severity at presentation, whereas our patients were systematically identified following denosumab discontinuation [1].

Treatment during over 3 years of follow-up post MVF was very variable: patients were prescribed denosumab, teriparatide, oral, and IV bisphosphonates in different sequences. A third (2/6) of the patients who received denosumab following MVF, discontinued treatment after a second treatment sequence.

Since a rebound increase in bone turnover rate is assumed to be the underlying mechanism for MVF after denosumab discontinuation, a timely bone resorption inhibition by reinstituting denosumab is perhaps the most appropriate treatment modality [21], although, according to one report, it may not completely eliminate the risk for recurrent vertebral fracture [36]. In our core MHS study, most of the cohort (1338/1500; 89\%) discontinued denosumab treatment administered before the end of 3 years [1]. This shortterm exposure time was interpreted as implying inadvertent patient dropout rather than planned physician-initiated discontinuation. If this is the case, subsequent unintentional treatment dis- 
continuations, or incompliance, are equally likely and were indeed demonstrated in this sub-group analysis, indicating that intensive patients' education should be included as an integral part of a comprehensive intervention for secondary prevention of osteoporotic fractures. Suboptimal compliance is further suspected by incomplete treatment course with teriparatide (P5, P7, P9, and P10) and lack of antiresorptive medication administration following teriparatide (P5).

Only four patients ( $33 \%$ ) of the denosumab discontinuers received bisphosphonates, either oral or IV, after the MVF event, even though bisphosphonates are the most commonly recommended treatment modality after denosumab discontinuation [21, 37]. A recently published systematic review and position statement by European Calcified Tissue Society (ECTS) recommends that patients with shorter duration of denosumab treatment (up to 2.5 years) and otherwise low fracture risk should receive treatment with an oral bisphosphonate for 1 to 2 years. Patients who have been treated with denosumab for a longer period or who are at persistently high risk for fracture should receive zoledronate [21].

Four (a third) of our denosumab discontinuers received teriparatide as a first medication after the MVF. As previously mentioned, rapid bone turnover suppression is considered the cornerstone of treatment of the rebound increase in bone turnover phenomena, while teriparatide exerts its action via stimulation of bone turnover [38]. It has been shown that women who received 2 years of denosumab and were switched to 2 years of teriparatide experienced a transient BMD decline [39]. One of our patients (P7) sustained MVF, after denosumab discontinuation and two months into teriparatide treatment. It has been already suggested that teriparatide would be undesirable following denosumab treatment $[21,40]$.

Two denosumab discontinuers underwent vertebroplasty/kyphoplasty, procedures previously shown, in several case reports, to bring about additional vertebral fractures upon denosumab discontinuation and are currently not recommended for the treatment of vertebral fractures in the scenario of denosumab discontinuation [35].

Two patients sustained recurrent vertebral fractures, one patient (P6) after additional denosumab discontinuation and the other, $\mathrm{P} 9$, was treated with teriparatide (though of the suboptimal duration), followed by denosumab and zoledronic acid. Patient-related factors as well as vulnerability of the already-fractured spine could have accounted for the scenario of the second patient [41].

Over 36.5 months of follow-up, two patients in the MVF-DD group passed-away (one of those was not treated following an MVF and another sustained recurrent vertebral fracture), reflecting the extreme fragility of some of the patients and the additional risk caused by recurrent fractures.

Our study has several limitations. Due to the retrospective nature of secondary data collection from an HMO database, rather than from personal interaction with the patients, we were unable to ascertain the rationale for the clinical decisions regarding the management after denosumab discontinuation. Since reports on vertebral fractures after denosumab discontinuation emerged after 2015 , it is conceivable that some laxity in the management of denosumab discontinuers, which in our patients mainly occurred between 2015-2017, could have been affected by the lack of clear practice guidelines regarding this condition at that time. Finally, a core study design, specifically the definition of discontinuation as a 3-month (and not less) refill gap and the follow-up for fractures post discontinuation as one year (and not more) might have caused underestimation of fracture incidence and a selection bias, since patients presenting before or after this time-frame may also have fractures related to denosumab discontinuation, with distinct characteristics.

The strengths of our report lay in the systematic methodology of data retrieval from an established, high-quality, database, meticulous adjudication of the MVF events, and unique long-term follow-up (>3 years) after MVF.

It should be noted, that the 2017 ECTS position statement [37], recommends that physicians, patients, and regulatory authorities should be educated on the potential risk of discontinuing denosumab treatment and on measures that should be taken to prevent unscheduled treatment discontinuation. The 2019 Endocrine Society updated guidelines on pharmacological management of osteoporosis in postmenopausal women, highlights the need for alternative antiresorptive treatment upon denosumab discontinuation [42]. The 2020 updated version of the ECTS position statement on fracture risk and management of discontinuation of denosumab therapy [21], the authors further stress the need for prompt intervention to reduce high bone turnover in patients who sustain vertebral fracture following denosumab discontinuation.

In conclusion, we presented a case series of real-world patients with MVF following denosumab discontinuation. The long-term follow-up reveals that management is inconsistent, and recurrent fractures are not uncommon. It calls for special attention to this high-risk group and implementation of management guidelines for patients with MVF after denosumab discontinuation [21].

\section{Conflict of Interest}

The core study [1] was investigator-initiated and was supported by Amgen. The current analysis was not sponsored. Dr. Tripto-Shkolnik reports personal fees from Amgen, personal fees from Eli Lilly, outside the submitted work. Prof. Yair Liel, Naama Yekutiel and Dr. Inbal Goldshtein, have nothing to disclose.

\section{References}

[1] Tripto-Shkolnik L, Fund N, Rouach V et al. Fracture incidence after denosumab discontinuation: Real-world data from a large healthcare provider. Bone 2020; 130: 115150

[2] Cummings SR, Martin JS, McClung MR et al. Denosumab for prevention of fractures in postmenopausal women with osteoporosis. N Engl J Med 2009; 361: 756-765

[3] Bone HG, Wagman RB, Brandi ML et al. 10 years of denosumab treatment in postmenopausal women with osteoporosis: Results from the phase 3 randomised FREEDOM trial and open-label extension. Lancet Diabetes Endocrinol 2017; 5: 513-523. http://linkinghub. elsevier.com/retrieve/pii/S2213858717301389

[4] Lewiecki EM, Miller PD, McClung MR et al. Two-year treatment with denosumab (AMG 162) in a randomized phase 2 study of postmenopausal women with low BMD. J Bone Miner Res 2007; 22: 1832-1841

[5] McClung MR. Denosumab in postmenopausal women with low bone mineral density. N Engl J Med 2006; 354: 821-831 
[6] Bone HG, Bolognese MA, Yuen CK et al. Effects of denosumab treatment and discontinuation on bone mineral density and bone turnover markers in postmenopausal women with low bone mass. J Clin Endocrinol Metab 2011; 96: 972-980

[7] Brown JP, Roux C, Törring $O$ et al. Discontinuation of denosumab and associated fracture incidence: Analysis from the Fracture Reduction Evaluation of Denosumab in Osteoporosis Every 6 Months (FREEDOM) Trial. J Bone Miner Res 2013; 28: 746-752

[8] Lamy O, Gonzalez-Rodriguez E. Underestimation of vertebral fractures after denosumab discontinuation. J Bone Miner Res 2018; 33: 547

[9] Lamy O, Gonzalez-Rodriguez E, Stoll D et al. Severe rebound-associated vertebral fractures after denosumab discontinuation: Nine clinical cases report. J Clin Endocrinol Metab 2016; 102: 354-358

[10] Popp AW, Zysset PK, Lippuner K. Rebound-associated vertebral fractures after discontinuation of denosumab - from clinic and biomechanics. Osteoporos Int 2016; 27: 1917-1921

[11] Polyzos SA, Terpos E. Clinical vertebral fractures following denosumab discontinuation. Endocrine 2016; 54: 271-272

[12] Aubry-Rozier B, Gonzalez-Rodriguez E, Stoll D et al. Severe spontaneous vertebral fractures after denosumab discontinuation: Three case reports. Osteoporos Int 2016; 27: 1923-1925

[13] Anastasilakis AD, Makras P. Multiple clinical vertebral fractures following denosumab discontinuation. Osteoporos Int 2016; 1929-1930

[14] Trovas G. Letter to the Editor: Severe rebound-associated vertebral fractures after denosumab discontinuation: Nine clinical cases report. J Clin Endocrinol Metab 2017; 102: 3781

[15] Anastasilakis AD, Polyzos SA, Makras P et al. Clinical Features of 24 Patients With Rebound-Associated Vertebral Fractures After Denosumab Discontinuation: Systematic Review and Additional Cases. J Bone Miner Res 2017; 32: 1291-1296

[16] Tripto-Shkolnik L, Rouach V, Marcus Y et al. Vertebral fractures following denosumab discontinuation in patients with prolonged exposure to bisphosphonates. Calcif Tissue Int 2018; 103: 44-49

[17] Anastasilakis AD, Yavropoulou MP, Makras P et al. Increased osteoclastogenesis in patients with vertebral fractures following discontinuation of denosumab treatment. Eur J Endocrinol 2017; 176: 677-683

[18] Cummings SR, Ferrari S, Eastell R et al. Vertebral fractures after discontinuation of denosumab: A post hoc analysis of the randomized placebo-controlled FREEDOM trial and its extension. J Bone Miner Res 2018; 33: 190-198

[19] Uebelhart B, Rizzoli R, Ferrari SL. Retrospective evaluation of serum CTX levels after denosumab discontinuation in patients with or without prior exposure to bisphosphonates. Osteoporos Int 2017; 28: 2701-2705

[20] Anastasilakis AD, Yavropoulou MP, Makras P. Bisphosphonates or denosumab discontinuation and risk of fractures. Maturitas 2017; 102: 75

[21] Tsourdi E, Zillikens MC, Meier $C$ et al. Fracture risk and management of discontinuation of denosumab therapy: A systematic review and position statement by ECTS. J Clin Endocrinol Metab. 2020; doi: $10.1210 /$ clinem/dgaa756 Online ahead of print

[22] Horne AM, Mihov B, Reid IR. Bone loss after romosozumab/denosumab: Effects of bisphosphonates. Calcif Tissue Int 2018; 103: 55-61

[23] Kendler D, Chines A, Clark P et al. Bone mineral density after transitioning from denosumab to alendronate. J Clin Endocrinol Metab 2020; 105: e255-e264

[24] Anastasilakis AD, Papapoulos SE, Polyzos SA et al. Zoledronate for the prevention of bone loss in women discontinuing denosumab treatment. A prospective 2-year clinical trial. J Bone Miner Res 2019; 34: $2220-2228$
[25] Goldshtein I, Chandler J, Shalev V et al. Osteoporosis in the community: findings from a novel computerized registry in a large health organization in Israel. jarlife.net. http://www.jarlife.net/download. html? type $=$ pdf\&id $=262$

[26] Gonzalez-Rodriguez E, Aubry-Rozier B, Stoll D et al. Sixty spontaneous vertebral fractures after denosumab discontinuation in 15 women with early-stage breast cancer under aromatase inhibitors. Breast Cancer Res Treat 2020; 179: 153-159

[27] Anagnostis P, Paschou SA, Gonzalez-Rodriguez E et al. Spontaneous Vertebral Fractures in Males with Osteoporosis After Denosumab Discontinuation. JCR J Clin Rheumatol 2019; doi: 10.1097/ RHU.0000000000000979 Online ahead of print

[28] Ebeling PR. Osteoporosis in men. N Engl J Med 2008; 358: 1474-1482

[29] Cavalier $E$, Bergmann $P$, Bruyère $O$ et al. The role of biochemical of bone turnover markers in osteoporosis and metabolic bone disease: a consensus paper of the Belgian Bone Club. Osteoporos Int 2016; 27: 2181-2195

[30] Nieves JW, Formica C, Ruffing J et al. Males have larger skeletal size and bone mass than females, despite comparable body size. J Bone Miner Res 2005; 20: 529-535

[31] Vestergaard P, Rejnmark L, Mosekilde L. Osteoporosis is markedly underdiagnosed: A nationwide study from Denmark. Osteoporos Int 2005; 16: 134-141

[32] Camacho PM, Petak SM, Binkley N et al. American Association of Clinical Endocrinologists/American College of Endocrinology Clinical Practice Guidelines for the Diagnosis and Treatment of Postmenopausal Osteoporosis- 2020 Update Executive Summary. Endocr Pract 2020; 26: 564-570

[33] Cummings SR, Melton L], Osteoporosis I. Epidemiology and outcomes of osteoporotic fractures. Lancet 2002; 359: 1761-1767

[34] Liel Y, Plakht Y, Tailakh MA. Bone turnover in osteoporotic women during long-term oral bisphosphonates treatment: Implications for treatment failure and "drug holiday" in the real world. Endocr Pract 2017; 23: 787-793

[35] Lamy O, Stoll D, Aubry-Rozier B et al. Stopping denosumab. Curr Osteoporos Rep 2019; 17: 8-15

[36] Niimi R, Kono T, Nishihara A et al. Second rebound-associated vertebral fractures after denosumab discontinuation. Arch Osteoporos 2020; $15: 7$

[37] Tsourdi E, Langdahl B, Cohen-solal M et al. Discontinuation of Denosumab therapy for osteoporosis : A systematic review and position statement by ECTS. Bone 2017; 105: 11-17

[38] Kraenzlin ME, Meier C. Parathyroid hormone analogues in the treatment of osteoporosis. Nat Rev Endocrinol 2011; 7: 647-656

[39] Leder BZ, Tsai JN, Uihlein AV et al. Denosumab and teriparatide transitions in postmenopausal osteoporosis (the DATA-Switch study): Extension of a randomised controlled trial. Lancet 2015; 386: 1147-1155

[40] Leder BZ. Optimizing sequential and combined anabolic and antiresorptive osteoporosis therapy. JBMR Plus 2018; 2: 62-68

[41] Gehlbach S, Saag KG, Adachi JD et al. Previous fractures at multiple sites increase the risk for subsequent fractures: The global longitudinal study of osteoporosis in women. J Bone Miner Res 2012; 27: 645-653

[42] Eastell R, Rosen C], Black DM et al. Pharmacological management of osteoporosis in postmenopausal women: An endocrine society clinical practice guideline. J Clin Endocrinol Metab 2019; 104: 1595-1622 